\title{
Non destructive transient thermal evaluation of laminated composites : discrimination between delaminations thickness variations and multidelaminations
}

\author{
by A. BENDADA, D. MAILLET and A. DEGIOVANNI (*)
}

(") Laboratoire d'Energétique et de Mécanique Theorique et Appliquée, URA CNRS 875 ENSEM, 2 avenue de la Forét de Haye, F-54500 Vandoeuvre Les Nancy, France.

\begin{abstract}
Different types of anomalies, such as local thickness changes, single or multi-delaminations, can be detected in carbon epoxy laminates using photothermal stimulation and infrared thermography. Discrimination functions, that allow to sort out the different types of the above-mentioned defects are constructed.
\end{abstract}

\section{Nomenclature}

a diffusivity

c specific heat

$e \quad$ thickness

$e_{d} \quad$ overthickness

$F \quad$ discrimination function

$k \quad(\mathrm{p} / \mathrm{a})^{1 / 2}$

$M \quad$ experimental Laplace transform (ELT) of contrast

$M^{\prime} \quad$ ELT of product t to contrast

$p \quad$ Laplace variable

$Q \quad$ energy surface density

$R_{c} \quad$ contact resistance

$t$ time

$T$ temperature

$u, v$ coefficients

$x$ depth $\alpha \quad$ square root of $\mathrm{p}^{*}$

$\delta \quad$ relative overthickness

$\lambda$ conductivity

$\rho \quad$ mass density

$\theta \quad$ Laplace transform of $\mathrm{T}$

Subscripts

eq equivalent

$f$ front side

$i$ delamination number

- sane of reference slab

$r$ rear side

$s \quad$ sensitivity coefficient

$\infty \quad$ asymptotic long time

Superscripts

$t \quad$ transposed

normalized or reduced

\section{Introduction}

The thermal non destructive evaluation technique presented here must detect and determine the nature of subsurface flaws in laminates such as carbon-epoxy slabs. In a very classical way [1], derived from the heat pulse method for thermal diffusivity measurement, one of the sides (front) of the laminate is stimulated using a heat pulse source while perturbations of the transient temperature field; produced by the presence of a possible defect, are analysed on the same or other side (rear) of the material.

In that types of laminates the defects the most commonly met are : inserts, delaminations, micro-porosity, local variation of the resin concentration, local variation of the thickness of the part and multidelamination (see figure 1).

Usually measurement of the detected defect can be considered only after its parameters (size, location, extent...) have been translated into thermal parameters, which means that the defect has been characterized, with respect to the particular experiment, and after the corresponding direct thermal problem has been solved. But, in order to select the right model that can be used for defect measurement, its nature has to be determined first. 
Criteria, based on discrimination functions, that are based on a one-dimension transfer model, are presented here : they allow to bring an answer to this very important qualitative problem.

It will be later shown that when a multi-delamination can be characterized by a succession of thermal contact resistances, of very low value compared to the sane slab total resistance, its response (contrast) corresponds to simple addition of the responses caused by each delamination considered separately.

Finally the possible homogeneization of a multidelamination with the increase of the number of delaminations will be studied.

\section{Local change of thickness and thermal contrast}

\subsection{Perspective of the problem}

Direct measurement of a local thickness variation can be difficult if the over (or under) thickness is located on a non- accessible side of the laminate (see figure 1.) This local overthickness can be charaterized by a reduced parameter $\delta$ that is the relative thickness change $e_{d}$ with respect to the reference slab thickness $e_{0}$ (positive in case of a bump and negative for a depression). If the thickness change occurs over a wide enough part of the slab, two fictitious one-dimensional $(1 D)$ heat pulse experiments can be considered to obtain the front side thermograms -temperature $T$ versus time $t$ curves- at the level of either this thickness change or the reference thickness. Information regarding the estimation of $\delta$ comes from the contrast thermogram, that is the difference of these two thermograms that have been normalized.

\subsection{Direct model}

The first heat pulse experiment that is considered corresponds to a slab of thickness $e=e_{0}+e_{d}$, with uniform initial temperature $T=0$ and absorbed energy density $Q$, the heat losses are neglected. The front side Laplace transform $\theta$ of temperature $T$ is:

$$
\theta=Q \operatorname{coth}\left[(1+\delta) e_{o}\right] /(\lambda k) \quad \text { with: } k^{2}=p / a \quad \text { (p:Laplace variable) }
$$

A similar expression can be obtained for the front side Laplace temperature $\theta_{0}$ of the second experiment on the reference slab of thickness $e_{o}$, replacing $Q$ by $Q_{O}$ and $\delta$ by zero. There is no reason to have uniform energy absorption in the real unique experiment shown in figure 1 , which means that $Q$ and $Q_{0}$ are different in the previous two fictitious experiments and normalization must be done with respect to the asymptotic temperatures $T_{\infty}$ and $T_{\text {oos. }}$ A contrast that cancels out for long times is therefore constructed :

$$
\begin{aligned}
& \Delta T^{*}=\frac{T}{T_{\infty}}-\frac{T_{0}}{T_{O \infty}}=T^{*}-T_{o}^{*} \\
& \text { with: } \quad T_{\infty}=Q /\left[\rho c e_{0}(1+\delta)\right] \quad \text { and } \quad T_{O \infty}=Q_{0} /\left(\rho c e_{0}\right)
\end{aligned}
$$

A reduced Laplace transform of the normalized temperature $T^{*}$ is defined and can be related to $\theta$ given in equation (1):

$$
\theta^{* *}=\int_{0}^{\infty} \exp \left(-p^{*} t^{*}\right) T^{*} d t^{*}=\lambda \theta /\left[Q e_{O}(1+\delta)\right]
$$

where integration is done using the reduced time or Fourier number $t^{\star}=a t / e^{2}$ that is associated with the reduced Laplace variable $p^{*}=e^{2} p / a$.

A similar expression $\theta_{0}^{* *}$ can be obtained for the reference experiment, replacing $T^{*}$ by 


\section{http://dx.doi.org/10.21611/qirt.1992.033}

$T_{0}^{*}, t^{*}$ by $t_{0}^{*}=(1+\delta)^{2} t^{*}$ and therefore $p^{*}$ by $p_{0}^{*}=p^{*} /(1+\delta)^{2}$ in equation (4).

Equations (3) written for the two experiments and the use of a common reduced time (common $p_{\mathrm{o}}^{*}$ ) allow the calculation of the reduced transform of equation (4), the Laplace contrast $\Delta \theta^{* *}$ :

$$
\Delta \theta^{* *}\left(p_{0}^{*}, \delta\right)=\frac{1}{\alpha_{O}}\left\{(1+\delta) \operatorname{coth}\left[(1+\delta) \alpha_{0}\right]-\operatorname{coth}\left(\alpha_{0}\right)\right\} \quad \text { with: } \alpha_{O}=\left(p_{0}^{*}\right)^{1 / 2}
$$

The original $\Delta T^{*}\left(t_{0^{*}}^{*}, \delta\right)$ of $\Delta \theta^{* *}\left(p_{0^{*}}^{*} \delta\right)$ is calculated using a numerical inversion algorithm developed by STEHFEST[3].

\subsection{Contrast and sensitivity}

In any parameters estimation problem, a very useful insight into the possibility of identifying a parameter $\delta$ is to plot the variation of its sensitivity coefficient versus the variable, here the Fourier number $t_{0}^{*}$. This sensitivity coefficient is simply the partial derivative of the signal, the contrast $\Delta T^{*}$ here, with respect to the parameter, multiplied by $\delta$ :it is plotted, together with the contrast signal $\Delta T^{*}$ in figure 2 for $\delta=+0.1$.

The contrast is a decreasing function of time; it goes to infinity for short times, showing a strong departure from delamination contrasts (see figure 2). The sensitivity coefficient and the contrast curves are very close together. At any time a $10 \%$ increase of $\delta$ produces a $10 \%$ increase of the contrast, making therefore the estimation of $\delta$ possible.

\subsection{Analytical expression for the overthickness}

The Laplace transform of the experimental contrast $\Delta T^{*}$ (equation (4)) can be calculated through time integration. Let us call $M$ this estimation of $\Delta \theta^{* *}\left(p_{0}^{*}, \delta\right)$. Once $M$ known, equation (5)- constitutes a transcendental equation. This equation can be iteratively solved, but an analytical solution can be obtained if equation (5) is derived with respect to $p_{o}^{*}$; after some manipulation on the two equations, one finds:

$$
\delta=1-\left[1-2 \alpha_{0}^{2} M^{\prime}+M+2 \alpha_{0} M \operatorname{coth}\left(\alpha_{0}\right)+\alpha_{o}^{2} M^{2}\right]^{1 / 2}
$$

with: $M=\int_{0}^{\infty} \exp \left(-p_{0}^{*} t_{0}^{*}\right) \Delta T^{*} d t_{0}^{*} \quad$ and $\quad M^{\prime}=\int_{0}^{\infty} \exp \left(-p_{0}^{*} t_{0}^{*}\right) \Delta T^{*} t_{0}^{*} d t_{0}^{*}$

One can notice that $M^{\prime}$, that is the opposite of the derivative of $M$ with respect to $p_{o}{ }_{0}$, can be experimentally estimated in a similar way as $M$.

\section{Multidelamination}

If the composite slab is submitted to a shock, the resulting delamination is seldom alone : one has therefore to consider the case of a multidelamination where each of the $n$ delaminations is characterized by a contact thermal resistance $R_{c i}$ located at a depth $x_{i}$ under the stimulated side (see figure 1).

In the case of a double delamination $(n=2)$, the Laplace contrasts for the two sides of the slab can be calculated, as in paragraph 2.2, using the quadripole method. If the $R_{C i}$ s are 
small compared to the sane slab total resistance $\left(R_{c i}^{*}=R_{c i} \lambda / e<1\right)$, a first order series expansion gives ( for $i=1$ or 2 and $x_{j}^{*}=x_{i} / e_{0}$ )

$$
\begin{aligned}
& \Delta \theta_{i}^{* *}=\left(R_{c 1}^{*} u_{1}+R_{c 2}^{*} u_{2}\right) / \sinh ^{2}\left(\alpha_{0}\right) \text { and } \Delta \theta_{r}^{* *}=-\left(R_{c 1}^{*} v_{1}+R_{c 2}^{*} v_{2}\right) / \sinh ^{2}\left(\alpha_{O}\right) \\
& \text { with: } \quad u_{i}=\sinh ^{2}\left[\alpha_{O}\left(1-x_{i}^{*}\right)\right] \quad \text { and } \quad v_{i}=\sinh \left(\alpha_{O} x_{i}^{*}\right) \sinh \left[\alpha_{O}\left(1-x_{i}^{*}\right)\right]
\end{aligned}
$$

One immediately notices that the contrasts caused by each single delamination simply adds up because of the linearity in $\left(R_{c 1}^{*}, R_{c 2}^{*}\right)$. This property is visualized in figure 3 for front side detection.

Even in the case of small contact resistances, equation (7) shows that an equivalent unique delamination $\left(R_{C}^{*}, x^{*}\right)$, that would produce the same contrast as the double delamination for the two sides, does not exist since its two parameters would depend on $p_{0}^{*}$ and therefore on time. This point can be underscored in figure 4 where the front side contrast caused by a double delamination is compared with the contrast of a unique delamination that produces both the same maximum level and the same sane slab Fourier number of its maximum -see [4]. One notices that the two contrasts are the same for short time but diverge for long times after the maximum. Even if the two contrasts have similar bell shape, their discrimination deserves to be considered.

\section{Discrimination function for a thickness variations : application to delaminations}

Figures 5 and 6 show front side contrasts caused by a single delamination, an overthickness and a depression. Their shape is completely different, which allows a qualitative discrimination.

A more quantitative discrimination is also possible : if equation (6) is written for two values $p_{01}^{*}$ and $p_{02}^{*}\left[\alpha_{01}=\left(p_{01}^{*}\right)^{1 / 2,} \alpha_{02}=\left(p_{02}^{*}\right)^{1 / 2}\right]$, elimination of $\delta$ leads to a function $F$ :

$$
F\left(M_{1}, M_{2}, M_{1}^{\prime}, M_{2}^{\prime}\right)=\sum_{i=1}^{2}(-1)^{i+1}\left[1-2 \alpha_{o i}^{2} M_{i}^{\prime}+M_{i}+2 \alpha_{o i} M_{i} \operatorname{coth}\left(\alpha_{o i}\right)\right]^{1 / 2}
$$

that cancels out for an overthickness (or depression) and that takes non-zero values for delaminations.Calculations of this function were done using Laplace transforms $M_{i}$ and $M_{i}^{\prime}$ produced by a single delamination for various reduced contact resistances $R_{c}^{*}$ located at various reduced depths $x^{*}$. Results of these simulations are presented in figure 7 for $p_{o 1}^{*}=1$ and $p_{02}^{*}=4$.

The always positive values of this function seem to qualify it as an overthickness discrimination function even if the effects of uncertainties in experimental estimations of its arguments remain to be studied.

A similar approach is possible for a single delamination where a corresponding discrimination function can be constructed using the expression of the reduced depth $x^{*}$ for front side detection (instead of $\delta$ ).

\section{Multidelamination and homogeneous laminate}

If the number of delaminations becomes large, the laminate behaves as a homogeneous 


\section{http://dx.doi.org/10.21611/qirt.1992.033}

material in terms of its rear-side thermogram [6]. Figure 8 shows the normalized rear-side thermogram of a carbon-epoxy laminate $(30 \%$ resin rate) containing $n$ equidistant delaminations of total thermal contact resistance $R_{C}$ equal to the sane slab resistance $(e / \lambda)$ and plotted versus the Fourier number constructed using the equivalent diffusivity $a_{e q}$ :

$$
a_{e q}=\lambda_{\theta q} /(\rho c) \quad \text { with }: e / \lambda_{e q}=e / \lambda+R_{C}
$$

Homogeneization is particularly slow since about 70 delaminations are necessary to see this thermogram meet the classical response of the homogeneous slab.

\section{Conclusion}

Non destructive thermal evaluation based on the technique of Laplace transforms of the locally normalized contrast not only allows the estimation of the parameters of detected defects but is also able to qualitatively discriminate between different possible explanations for an anomaly in the infrared frames. This model selection role can be played by the discrimination functions. These functions remain to be tested, using both simulation and experiments, in order to evaluate the influence of the measurement noise, of heat losses effects and of two or three-dimensional effects to determinate their limits.

\section{REFERENCES}

[1] MAILLET (D.), DIDIERJEAN (S.), HOULBERT (A.S.) and DEGIOVANNI (A.). Non destructive transient thermal evaluation of delaminations inside a laminate : a thermal processing technique of thermal images,. Quantitative Infrared Thermography, Eurotherm Seminar 27, Paris, July 7-9, 1992.

[2] DEGIOVANNI (A.). - Conduction dans un mur multicouche avec sources : extension de la notion de quadripôle. Int. J. Heat Mass Transfer, 31,3, 1988, p.553-557.

[3] STEHFEST (H.). -Remarks on algorithm 368. Numerical inversion of Laplace transforms. Com. A.C.M., 13, 1970, p.624.

[4] LAMINE (A.S.). - Caractérisation de défauts dans les matériaux composites par thermographie infrarouge. Thèse INPL Nancy, nov. 1988.

[5] DEGIOVANNI (A.). - Systèmes multicouches : problèmes liés à l'homogénéisation des caractéristiques thermiques, Revue Générale de Thermique, n`339, 1990, p.117-128.

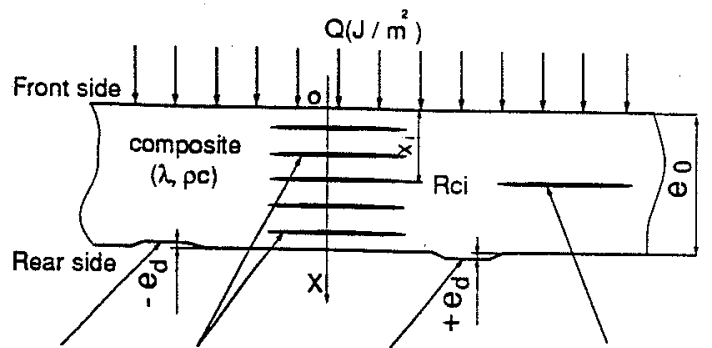

Cavity Multidelamination Overthickness Single-delamination

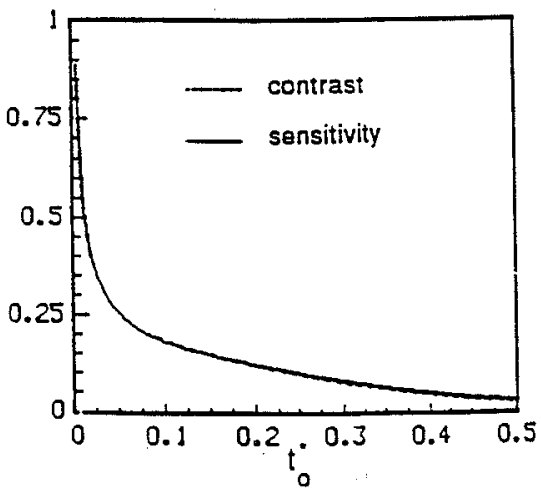

Fig.2.-Contrast on front side caused by an overthickness.Sensitivity to a relativo thickness variation $(\delta=+0.1)$ of the slab. 


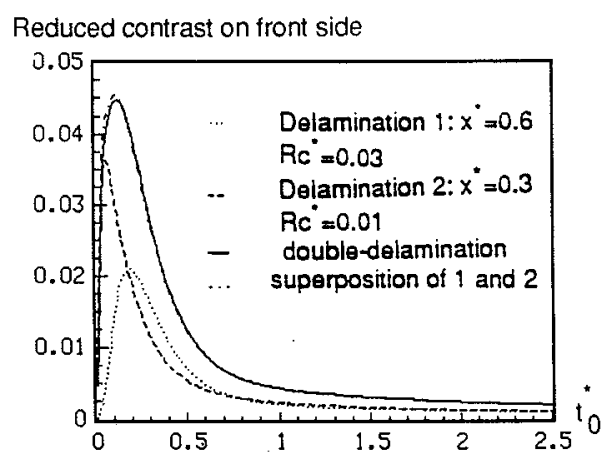

Fig.3.-Contrast superposition for delaminations of negligible resistances.

Contrast

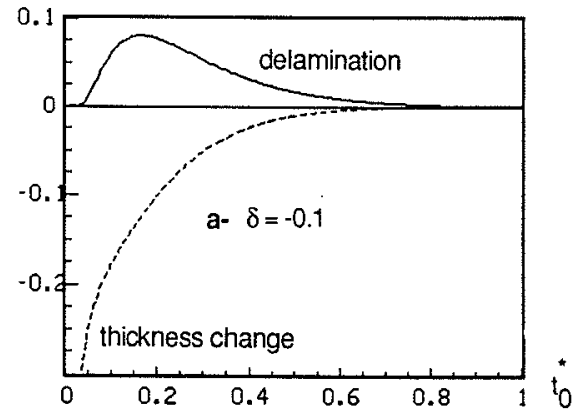

Contrast

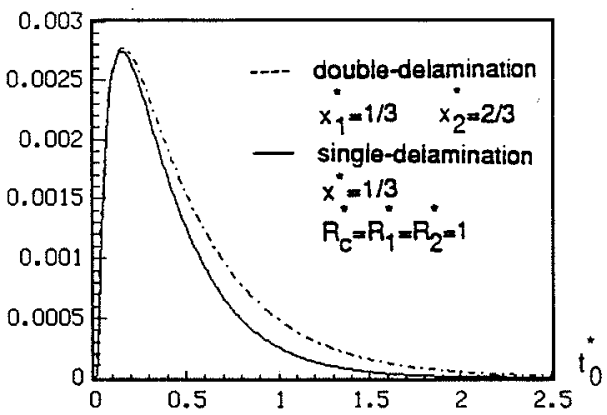

Fig.4.-Front side contrast of a delamination and a double delamination inside a carbone_epoxy slab.

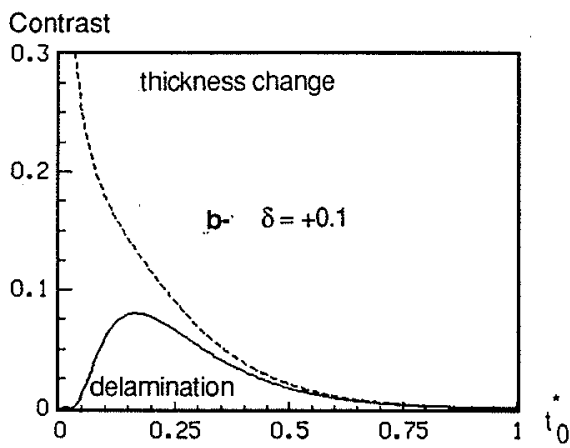

Fig.5 and 6.-Comparaison between a contrast caused by a mid_slab delamination ( $10 \%$ increase in thermal resistance of the slab) and a contrast caused by a relative thickness variation of the slab.

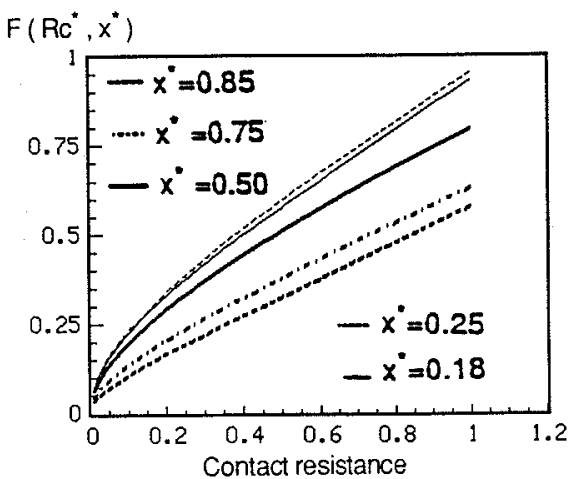

Fig.7.-Function of discrimination between two types of defects:delamination and thickness change.

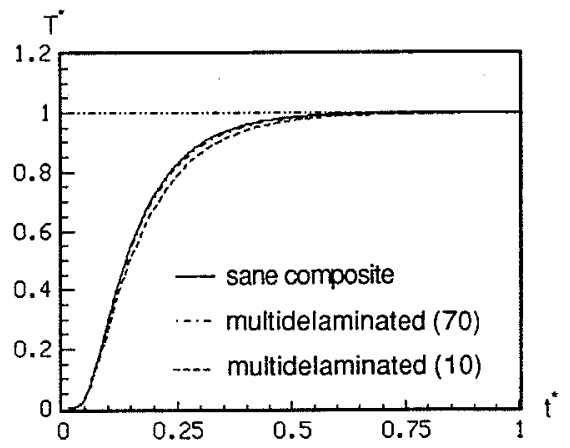

Fig.8.-Normalized thermograms on rear side for sane and multidelaminated samples $(n=10$ and 70 delaminations) 